\title{
Structural and molecular characteristics of axons in the long head of the biceps tendon
}

\author{
Roland Blumer $^{1}$ (D) Sandra Boesmueller ${ }^{2} \cdot$ Bernhard Gesslbauer $^{3} \cdot$ Lena Hirtler $^{1}$ • Daniel Bormann ${ }^{1}$. \\ Angel M. Pastor ${ }^{4} \cdot$ Johannes Streicher $^{5} \cdot$ Rainer Mittermayr $^{2}$
}

Received: 15 July 2019 / Accepted: 7 November 2019 / Published online: 7 December 2019

(C) The Author(s) 2019

\begin{abstract}
The innervation of the long head of the biceps tendon (LHBT) is not sufficiently documented. This is a drawback since pathologies of the LHBT are a major source of shoulder pain. Thus, the study aimed to characterize structurally and molecularly nervous elements of the LHBT. The proximal part of 11 LHBTs was harvested intraoperatively. There were 8 female and 3 male specimens. Age ranged from 66 to 86 years. For structural analyses, nervous elements were viewed in the transmission electron microscope. For molecular characterization, we used general neuronal markers including antibodies against neurofilament and protein gene product 9.5 (PGP9.5) as well as specific neuronal markers including antibodies against myelin basic protein (MBP), calcitonin gene-related product (CGRP), substance P (SP), tyrosine hydroxylase (TH), and growth-associated protein 43 (GAP43). Anti-neurofilament and anti-PGP9.5 visualized the overall innervation. Anti-MBP visualized myelination, antiCGRP and anti-SP nociceptive fibers, anti-TH sympathetic nerve fibers, and anti-GAP43 nerve fibers during development and regeneration. Immunolabeled sections were analyzed in the confocal laser scanning microscope. We show that the LHBT contains unmyelinated as well as myelinated nerve fibers which group in nerve fascicles and follow blood vessels. Manny myelinated and unmyelinated axons exhibit molecular features of nociceptive nerve fibers. Another subpopulation of unmyelinated axons exhibits molecular characteristics of sympathetic nerve fibers. Unmyelinated sympathetic fibers and unmyelinated nociceptive fibers express proteins that are found during development and regeneration. Present findings support the hypothesis that ingrowth of nociceptive fibers are the source of chronic tendon pain.
\end{abstract}

Keywords Tendon $\cdot$ Innervation $\cdot$ Nociceptive nerve fibers $\cdot$ Sympathetic nerve fibers $\cdot$ Developing nerve fibers

Roland Blumer and Sandra Boesmueller contributed equally to this work.

Roland Blumer

roland.blumer@meduniwien.ac.at

1 Center of Anatomy and Cell Biology, MIC, Medical University Vienna, A-1090 Vienna, Austria

2 AUVA Trauma Center Vienna Meidling, A-1120 Vienna, Austria

3 Department of Surgery, Division of Plastic and Reconstruction Surgery, Medical University Vienna, A-1090 Vienna, Austria

4 Departamento de Fisiología, Facultad de Biología, Universidad de Sevilla, 41012 Sevilla, Spain

5 Department of Anatomy and Biomechanics, Division of Anatomy and Developmental Biology, Karl Landsteiner University of Health Science, A-3500 Krems an der Donau, Austria

\section{Introduction}

A tendon is a though fibrous band that connects the muscle to the bone or muscle to muscle and is designed to transmit muscle force. Chronic overuse of tendons leads to tendon disorders (tendinopathy) which is associated with pain, swelling, and impaired mobility. Painful tendinopathy is a major clinical problem and associated with high socioeconomic costs because of sickness-induced nonproductive time. The etiology of chronic tendon pain often remains idiopathic.

Several studies have focused on nervous elements in tendons as the cause of chronic pain. Most commonly, neuroanatomical studies have been performed in the Achilles tendon due to frequency of tendinopathy not only in running and jumping sportsmen but also in sedentary and elderly persons (Alfredson and Lorentzon, 2000). Concordantly, these studies observed an increase of nerve fibers involving nociceptive fibers as well as sympathetic fibers in tendinopathic Achilles 
tendons compared to healthy tendons (Ackermann et al., 2003, Messner et al., 1999, Schubert et al., 2005). Most commonly nerve fibers are associated with blood vessels. In accordance, nociceptive and sympathetic fibers have been found in other tendons of the body including the proximal tendons of the forearm muscles (Ljung et al., 1999b, Ljung et al., 1999a, Ljung et al., 2004) and the patellar tendon (Danielson et al., 2008).

Alpantaki et al. (2005) were the first who analyzed the innervation of the long head of the biceps tendon (LHBT). They have reported that the LHBT contains a large network of nociceptive and sympathetic nerve fibers and that this neuronal net is not associated with blood vessels (Alpantaki et al., 2005). Only visual information of the general innervation was provided but no image data of the pattern of nociceptive and sympathetic innervation (Alpantaki et al., 2005). Different to Alpantaki et al. (2005), other studies on the LHBT showed that nerve fibers had a linear appearance and were close to vascular structures (Boesmueller et al., 2017, Singaraju et al., 2008, Blumer et al., 2019). However, while Singaraju et al. (2008) and Blumer et al. (2019) confirmed the occurrence of nociceptive nerve fibers in the LHBT, this type of nerve fiber has not been detected by Boesmueller et al. (2017). An increase of innervation was described in the paratendon of tendinopathic LHBTs without, however, providing qualitative information on nervous elements (Schmalzl et al., 2019). Aiming for the sympathetic innervation in LHBT, Tosounidis et al. (2013) failed to visualize sympathetic nerve fibers and instead described non-neural elements (tenocytes) displaying molecular features of sympathetic nerves. On the whole, the innervation of the LHBT is not sufficiently documented and discrepancies between different studies are apparent. To clarify pattern and origin of pain is significant because pathologies of the LHBT not only are common in overhead athletes but also affect non-recreational sportsmen and have been identified as a major source of shoulder pain (Ahrens and Boileau, 2007, Sethi et al., 1999). Thus, the present study was set out to analyze in detail structural and molecular characteristics of nervous elements in the LHBT.

\section{Material and methods}

\section{Tissue collection}

The LHBTs were harvested intraoperatively from patients suffering either from osteoarthritis (OA, $n=6)$ or a nonreconstructable head split fracture $(n=5)$ undergoing shoulder joint replacement. Eleven LHBTs were analyzed in this study. There were 8 female and 3 male specimens and age ranged from 66 to 86 years. For our analyses, we used the most proximal part of the LHBTs. Human spinal cord was harvested from an organ donor according to the special agreement signed by the individual before death and served as positive control for antibodies used in this study (Gesslbauer et al., 2017).

All procedures performed in this study were approved by the local medical ethics committee (Austrian workers compensation boards' research ethics committee and institutional review board of the Medical University of Vienna, No. 06/ 2017) and in accordance with the 1964 Helsinki declaration and its later amendments or comparable ethical standards. Informed consent was obtained from all persons included in this study.

\section{Tissue preparation}

Tendons were immersion fixed for $24 \mathrm{~h}$ at $4{ }^{\circ} \mathrm{C}$ in $4 \%$ paraformaldehyde in $0.1 \mathrm{M}$ phosphate buffer at $\mathrm{pH}$ 7.4. Thereafter, tissue was rinsed in phosphate-buffered saline (PBS) at $\mathrm{pH} 7.4$, cryo-embedded and kept at $-80{ }^{\circ} \mathrm{C}$ before further processing. A detailed description for cryo-embedding of tendons is provided by Blumer et al. (2019). Cryo-embedded tissue was used for immunofluorescence and analyzed in the confocal laser scanning microscope (CLSM) or slide scanner. Some cryo-embedded tendons were re-embedded in epon and analyzed in the transmission electron microscope.

\section{Immunofluorescence}

\section{Antibodies}

Different neuronal markers were used to determine the molecular characteristics of tendon innervation. The antibodies/ toxin used in the present study including working dilutions, PRID numbers, and suppliers are listed in Table 1.

\section{Immunolabeling}

Cryo-sections at $10 \mu \mathrm{m}$ thickness were cut and mounted on slides. To visualize different nervous qualities, a series of double labeling experiments were performed.

1. To visualize the overall innervation pattern, we used two general neuronal markers (anti-neurofilament and antiprotein gene product 9.5) and performed double labeling with antibodies against neurofilament and protein gene product 9.5 (PGP9.5). Phalloidin which binds to actin filaments was used to label smooth muscle cells in blood vessels. Phalloidin staining was combined with antineurofilament labeling.

2. For visualization of nociceptive fibers, we used antibodies against calcitonin gene-related peptide (CGRP) and substance $\mathrm{P}(\mathrm{SP})$ and performed three staining combinations including (i) antibodies against CGRP and neurofilament, 
Table 1 List of antibodies and toxins used in the present study including working dilution and suppliers

\begin{tabular}{llll}
\hline & Working dilution & PRID number & Supplier \\
\hline Primary antibodies & & & \\
Chicken anti-neurofilament & $1: 2000$ & AB_177520 & Merck/Millipore \\
Rabbit anti-protein gene product 9.5 & $1: 400$ & AB_2210632 & Merck/Millipore \\
Rat anti-myelin basic protein & $1: 500$ & AB_94975 & Merck/Millipore \\
Rabbit anti-calcitonin gene-related product & $1: 1000$ & AB_672958 & Merck/Millipore \\
Rabbit anti-growth-associated protein 43 & $1: 500$ & AB_109488 & Merck/Millipore \\
Rabbit anti-tyrosine hydroxylase & $1: 500$ & AB_390204 & Merck/Millipore \\
Mouse anti-substance P & $1: 500$ & AB_300971 & Abcam \\
Toxin & & & \\
Phalloidin AF488 & $1: 200$ & & Merck/Millipore \\
\hline
\end{tabular}

(ii) antibodies against SP and neurofilament, and (iii) antibodies against CGRP and SP.

3. To label sympathetic nerve fibers, we used antibody against tyrosine hydroxylase (TH) and performed double labeling with antibodies against $\mathrm{TH}$ and neurofilament.

4. To visualize nerve fibers during development and regeneration, we used antibody against growth-associated protein 43 (GAP43) and performed double labeling with (i) antibodies against GAP43 and neurofilament and (ii) antibodies against GAP43 and SP.

5. Finally, to visualize myelination, we used antibody against myelin basic protein (MBP) in the following staining combinations: (i) antibodies against MBP and neurofilament, (ii) antibodies against MBP and CGRP, (iii) antibodies against MBP and SP, (iv) antibodies against MBP and $\mathrm{TH}$, and (v) antibodies against MBP and GAP43.

Prior to labeling, frozen sections were dried at room temperature for $30 \mathrm{~min}$. Then tissue was blocked for $2 \mathrm{~h}$ in $10 \%$ normal goat serum in PBS containing $0.1 \%$ Triton (PBS-T). Thereafter, sections were incubated for $48 \mathrm{~h}$ with the primary antibodies, washed with PBS-T, and incubated for $2 \mathrm{~h}$ with AlexaFluor 488 and 568 conjugated secondary antibodies at concentration of 1:500. Finally, the tissue was rinsed again in PBS-T and coverslipped with fluorescence mounting medium.

Fluorescently labeled sections were analyzed with a confocal laser scanning microscope (CLSM (Olympus FV3000, Olympus Europa SE \& Co. KG, Hamburg, Germany)) or alternatively with a slide scanner microscope (Olympus VS120). In CLSM series of virtual sections of $0.9 \mu \mathrm{m}$ thickness were cut through the structures of interest. Each section was photo-documented with a $1024 \times 1024$-pixel resolution and $3 \mathrm{D}$ projections were rendered using the Image $\mathrm{J}$ software (NIH, Bethesda, MA, USA). Double-colored images were generated using lasers with excitation wavelength 488 and $568 \mathrm{~nm}$. Additionally, bright field images were recorded in the CLSM to match immunolabeled structures with the anatomical structure of the tendon.
For negative controls, primary antibodies were omitted and secondary antibodies used alone. In all cases, the omission of the primary antibody resulted in a complete lack of immunostaining. In positive controls, the validity of the antibodies was tested in the human spinal cord which was obtained from an organ donor (Gesslbauer et al., 2017).

\section{Transmission electron microscopy}

Some cryo-embedded tendons were thawed again and immersion fixed in modified Karnovsky solution containing 2\% paraformaldehyde and $2.5 \%$ glutaraldehyde in $0.1 \mathrm{M}$ PBS at $\mathrm{pH} 7.4$ for $24 \mathrm{~h}$ at $4{ }^{\circ} \mathrm{C}$. Following rinsing in PBS, tissue was postfixed in $1 \%$ osmium tetroxide in PBS for $12 \mathrm{~h}$ at $4{ }^{\circ} \mathrm{C}$, dehydrated in graded dilutions of ethanol, and embedded in epon. Semithin sections of epon-embedded tissue were cut with an Ultracut UCT (Leica, Wetzlar, Germany) and stained with toluidine blue and viewed under the light microscope. When structures of interest were detected at light microscopic level, ultrathin sections were cut, mounted on dioxaneformvar coated copper grids, immersed in an aqueous solution containing $2 \%$ uranyl acetate followed by a solution of $0.4 \%$ lead citrate, and examined under a transmission electron microscope Phillips 10 (Phillips, Amsterdam, Netherlands).

\section{Results}

\section{Specificity controls of the antibodies}

Human spinal cord was used as reference tissue to test the specificity of the antibodies (MBP, CGRP, SP, TH, and GAP43). The following double labeling experiments were performed: (i) anti-neurofilament/anti-MBP, (ii) anti-CGRP/ anti-SP, (ii) anti-neurofilament/anti-TH, and (iv) anti-neurofilament/anti-GAP43. Anti-neurofilament is a general marker for axons. Anti-MBP visualized myelinated axons (Kies et al., 1972), anti-CGRP and anti-SP nociceptive axons (Maggi, 1995), anti-TH adrenergic, sympathetic axons 
(Akesson et al., 1998), and anti-GAP43 axons during development and regeneration (Woolf et al., 1992).

Labeling with anti-neurofilament/anti-MBP showed that axons in the ventral and dorsal root of the spinal cord were encircled by a myelin sheath as demonstrated by MBP immunoreactivity (Fig. 1(a-a")). Labeling with anti-SP/anti-CGRP exhibited co-localization of CGRP/SP in superficial layer of the dorsal horn of the human spinal cord (Fig. 1(b-b")) in accordance with literature (Li et al., 2000). Double labeling with anti-neurofilament and anti-TH showed that arteries of the human spinal cord were surrounded by thin $\mathrm{TH}$ immunoreactive nerves (Fig. 1(c-c"c)) (Akesson et al., 1998). By labeling with anti-neurofilament/anti-GAP43, we did not expect GAP43 immunoreactivity in somatic axons of the adult human spinal cord. Exclusively, vegetative (sympathetic) nerve fibers around blood vessels exhibited GAP43
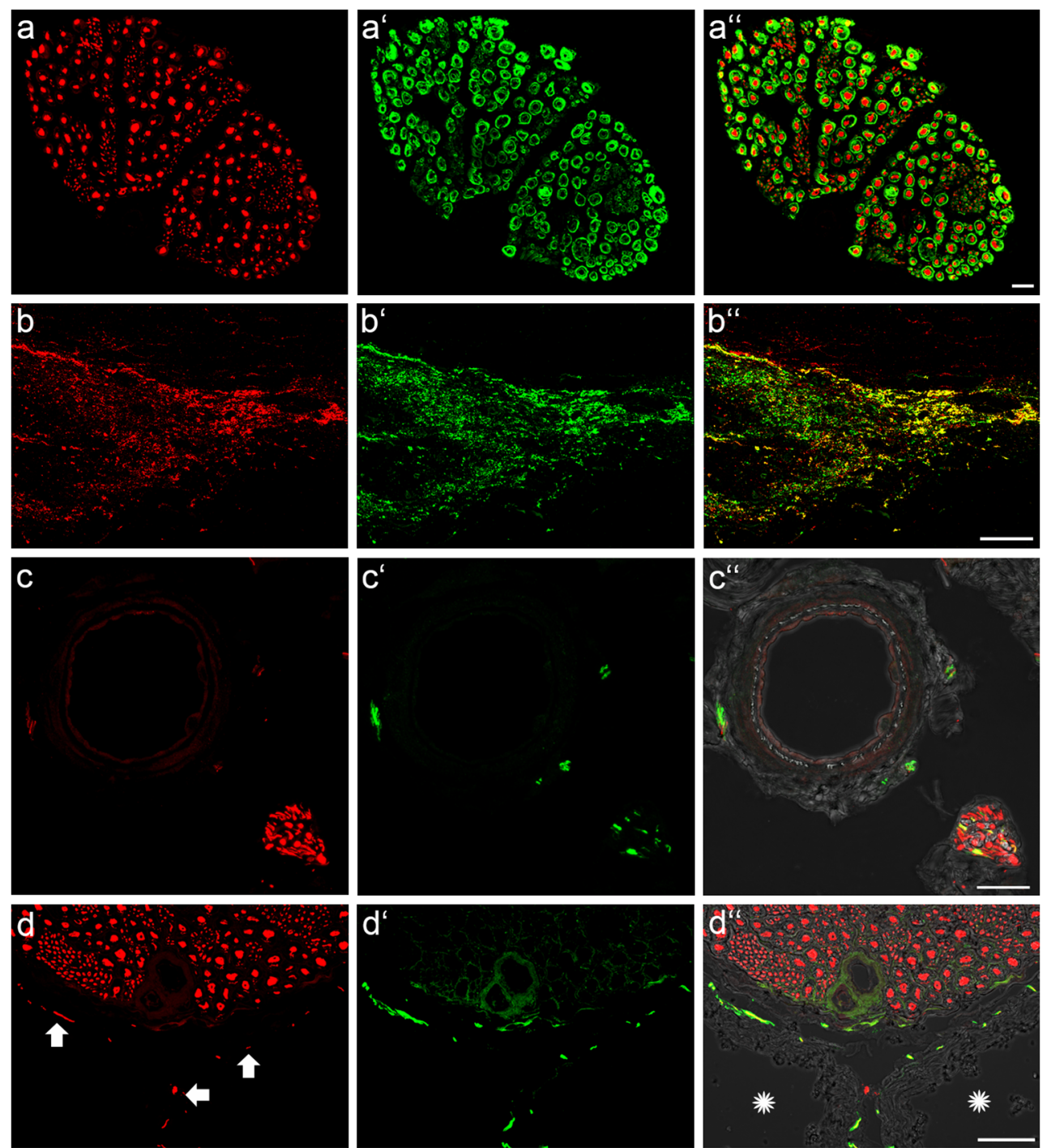

Fig. 1 CLSM images of immunofluorescently labeled cross section through the human spinal cord which served as reference tissue for the validity of the antibodies used in the present study. (a-a") Double labeling with anti-neurofilament (red) and anti-MBP (green). (a) Neurofilamentpositive nerve fibers of the dorsal root. ( $\mathrm{a}^{\mathrm{c}}$ ) Labeling of the myelin sheath by anti-MBP. (a"), Overlay of $\left(a, a^{6}\right)$ showing that axons are encircled by a myelin sheath. Scale bar, $20 \mu \mathrm{m}$. (b-b") Double labeling with anti-SP (red) and anti-CGRP (green). (b) SP and (b') CGRP immunoreactivity in the superficial layer of the dorsal horn. (b") Overlay of (b, b") exhibiting co-localization of CGRP and SP resulting in yellow mixed color. Scale bar, $100 \mu \mathrm{m}$. (c-c") Double labeling with anti-NF (red) and anti-TH (green). (c) Neurofilament-positive and (c') TH-positive nerve fibers. (c") The overlay of $\left(c, c^{c}\right)$ is merged with a bright field image which shows the anatomical structure of the artery. Nerve fibers in the wall of the artery are positive for NF and $\mathrm{TH}$. The nerve fascicle in the lower part of the image contains NF/TH-positive nerve fibers as well as axons lacking TH immunoreactivity (somatic axons). Scale bar, $20 \mu \mathrm{m}$. (d-d") Double labeling with anti-NF (red) and anti-GAP43 (green). (d) Neurofilament-positive nerve fibers of the white matter and outside the spinal cord (arrow). (d') GAP43 immunoreactivity is absent in somatic axons of the adult spinal cord. Only thin axons outside the spinal cord exhibit GAP43 immunoreactivity. The overlay of $\left(d, d^{\prime}\right)$ is merged with a bright field image which shows the anatomical structure of arteries (asterisks). Only thin axons (sympathetic axons) in the wall of arteries exhibit NF/GAP43 immunoreactivity. Scale bar, $50 \mu \mathrm{m}$ 
immunoreactivity (Fig. 1(d-d"s)) which is in accordance with literature (Woolf et al., 1992). In conclusion, immunostainings of the human spinal cord were in agreement with findings in literature and proved the specificity of the antibodies. Using these staining protocols, we subsequently analyzed the molecular signature of nervous elements in LHBT. Besides immunofluorescence, structural analyses of nervous elements were performed by high-resolution microscopy (transmission electron microscopy). Figure $2 \mathrm{a}$ shows the area of the LHBT analyzed in the present study.

\section{Axons are in the vicinity of blood vessels}

To visualize the overall innervation of the LHBT, we performed immunolabeling with two general neuronal markers, namely, anti-neurofilament and anti-PGP 9.5. Smooth muscle cells in blood vessels were counterstained with phalloidin.
Images were viewed with the slide scanner and the CLSM. In these and in other staining combinations, CLSM fluorescence images and bright field images were merged to map immunolabeling to the anatomical structures of the tissue.

Overview images from the whole cross-sectional area of the LHBT showed that neurofilament-positive nerve fibers were unevenly distributed within the tendon and areas with high axonal density alternated with areas devoid of axons (Fig. 2b, c). Double labeling with anti-neurofilament/anti-PGP9.5 confirmed that all axons were neurofilament/PGP 9.5 immunoreactive (Fig. 3(a-

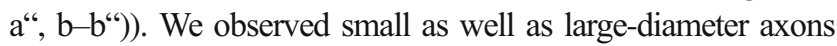
which had a linear appearance and were bundled in nerve fascicles of various dimensions (Fig. 3(a-acc, b-bc)). Nerve fascicles were in the vicinity of blood vessels but outside the adventitial layer of the vascular wall. Additionally, we observed blood vessels exhibiting single axons within the vascular wall while other closely located blood vessels were without innervation. To define
Fig. 2 Macroscopic view and fluorescent images of the long head of the biceps tendon (LHBT). a Macroscopic view of the proximal part of the LHBT. The inset indicates the region which was analyzed in the present study including the most proximal part of the tendon. Scale bar, $1 \mathrm{~cm}$. b Fluorescent image from the slide scanner showing a cross section through the whole tendon. Nerve fibers are labeled with antineurofilament. For higher contrast, the image is converted into gray and white colors and signals are enhanced which increases the background noise. Nerve fibers (arrows) appear as white dots and the background signal exhibits structural features of the tendon. Single nerve fibers group in fascicles and are unevenly distributed throughout the tendon. Fascicles are close to blood vessels. Scale bar, $1 \mathrm{~mm}$. c Inset of $\mathbf{b}$ showing nerve fascicles and associated blood vessels (bv) at higher magnification. Scale bar, $100 \mu \mathrm{m}$
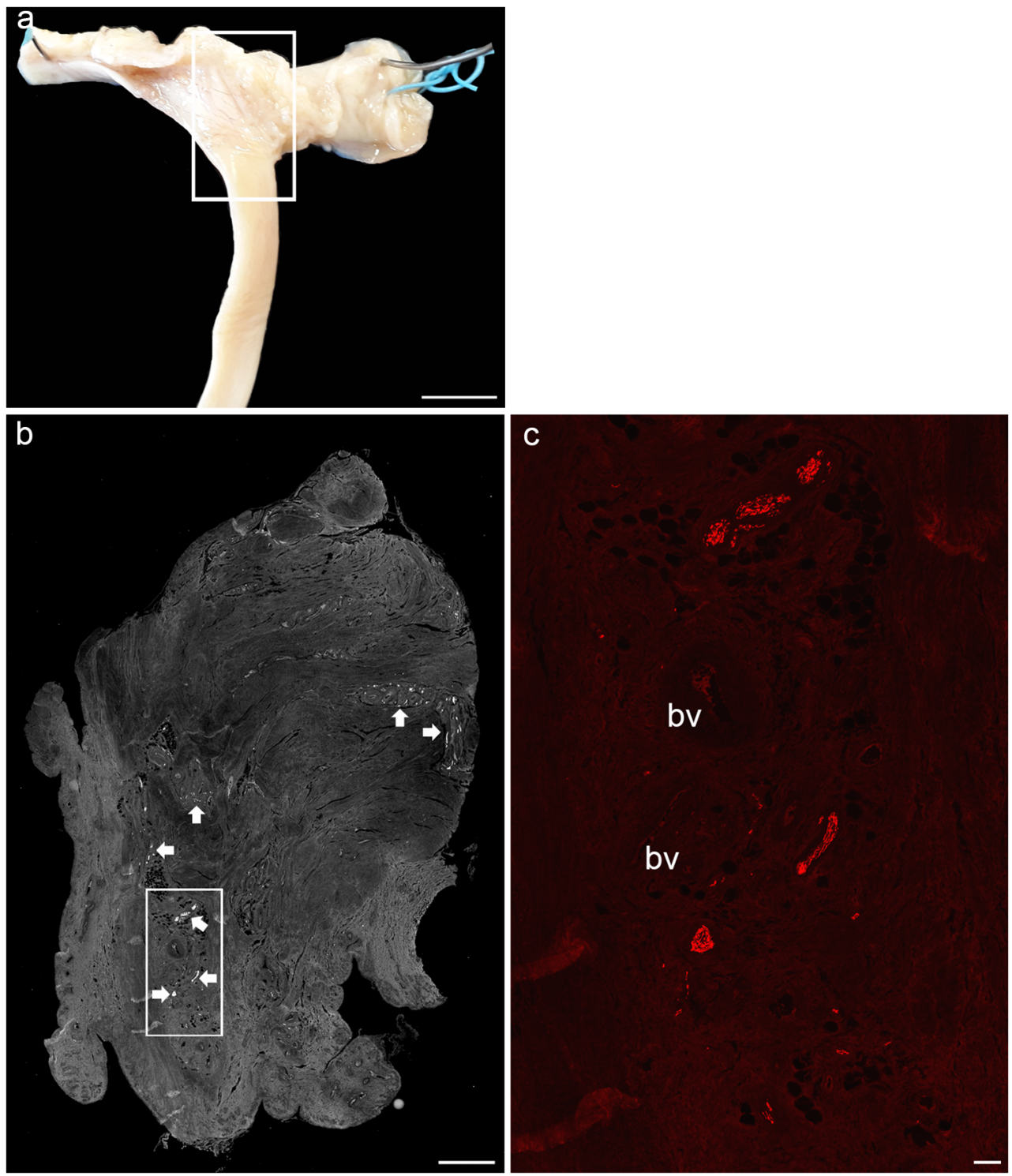
the type of blood vessel (artery versus vein) receiving innervation, we performed labeling with anti-neurofilament and phalloidin. Phalloidin binds to actin filaments of smooth muscle cells and the thickness of the muscle layer allowed us to distinguish between thick-walled arteries and thin-walled veins. Exclusively, arteries had single axons near the junction of the muscle and the adventitial layer. Veins completely lacked innervation (Fig. 3(c, d)).

\section{Unmyelinated and myelinated axons are present}

In the peripheral nervous system, small-diameter unmyelinated axons and large-diameter myelinated axons can be distinguished. Since axons in the LHBT varied in diameter, we hypothesized that different morphological types of axons (unmyelinated versus myelinated axons) are present in the LHBT. For axonal typing, we performed immunolabeling and fine structural analyses. Myelin was visualized using antibody against myelin basic protein (MBP). Nerve fibers were counterstained with anti-neurofilament. For structural evaluation, semithin sections of epon-embedded tendons were viewed in the light microscope. When axons were identified at the light microscopic level, ultrathin sections were cut and viewed in the transmission electron microscope.

Staining with anti-neurofilament/anti-MBP showed that many neurofilament-positive axons were MBP negative, whereas other neurofilament-positive axons were encircled by a myelin sheath as demonstrated by MBP immunoreactivity. Unmyelinated and myelinated axons were found in every LHBT (Fig. 4(a-a", b-b“)). Unmyelinated axons had a
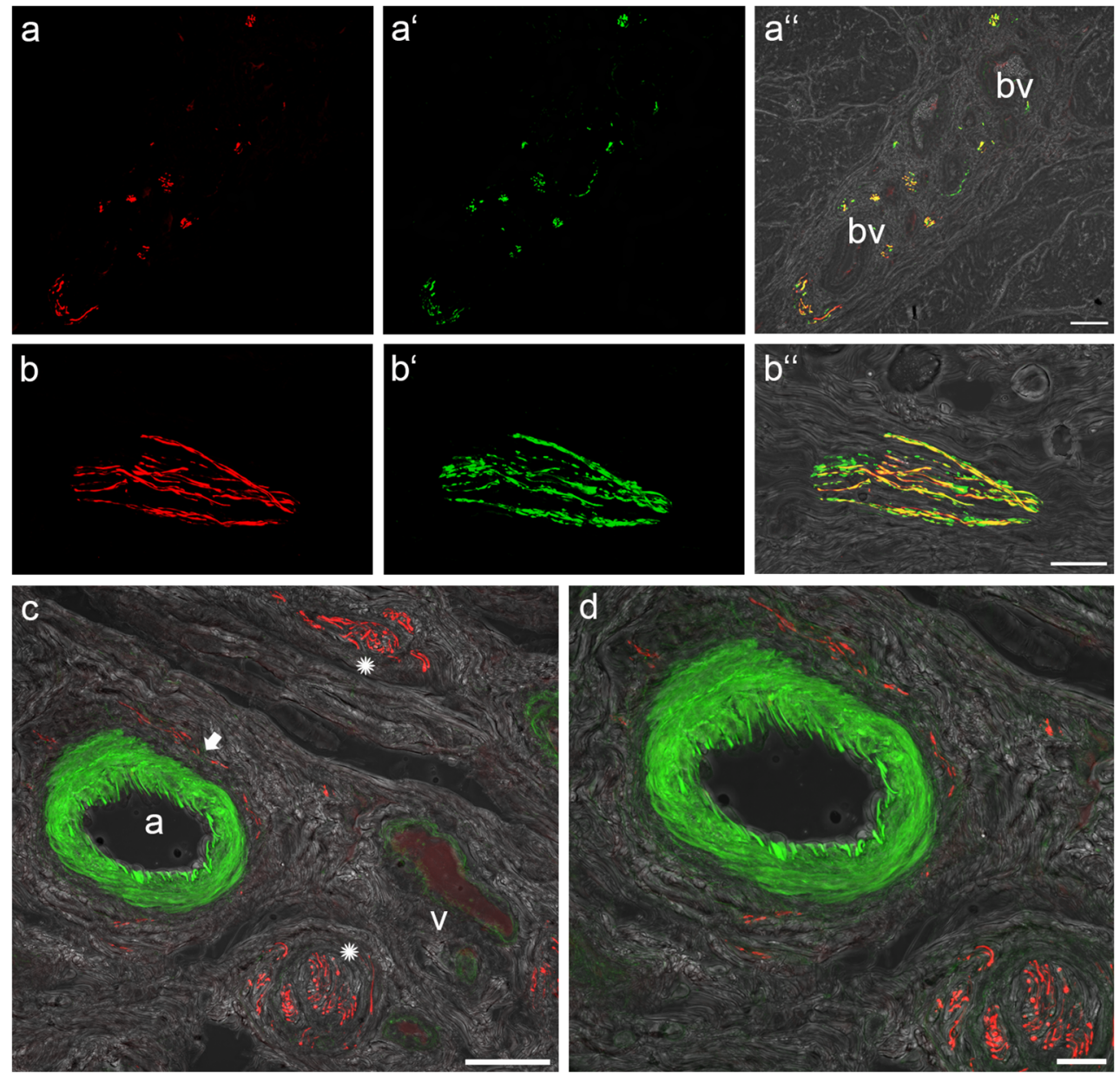

Fig. 3 CLSM images of sections labeled with two general neuronal markers (anti-neurofilament and anti-PGP9.5) and neurofilament and phalloidin. In these and other figures (Figs. 4, 6, 7, 8, and 9), fluorescence and bright field images are merged to map immunolabeling to anatomical structures. (a-a") orthogonal view and (b-b") longitudinal view of nerve fibers double labeled with anti-neurofilament (red) and anti-PGP9.5 (green). (a, b) Nerve fibers in neurofilament staining and $\left(a^{6}, b^{c}\right)$ PGP 9.5 staining. (a", b") Overlay showing complete overlap of neurofilament and PGP9.5 resulting in yellow mixed color. The bright field view shows the anatomical structure of blood vessels (bv) and collagen fibers. Blood

vessels are surrounded by immunoreactive nerve fascicles (a"). Collagen fibers are around the nerve fibers $\left(\mathrm{b}^{\prime \prime}\right)$. Scale bars, $50 \mu \mathrm{m}\left(\mathrm{a}-\mathrm{a}^{\mathrm{a}}\right)$ and $25 \mu \mathrm{m}$ (b-b"). (c, d) Double labeling with anti-neurofilament (red) and phalloidin (green). (c) Two nerve fascicles (asterisks) in the vicinity of an artery (a) and a vein (v). The muscular layer of the blood vessels visualized by phalloidin staining is much more pronounced in the artery. In the artery, single axons (arrow) are at the junction of the muscular and the adventitial layer. The vein lacked innervation. (d) The artery and nerve fibers at high magnification. Scale bars, $50 \mu \mathrm{m}$ (c) and $20 \mu \mathrm{m}$ (d) 
smaller-diameter than myelinated axons. The ratio of unmyelinated to myelinated axons varied within nerve fascicles. Specifically, we observed nerve fascicles where the majority of the axons were endowed with a myelin sheath and other fascicles where only a minority of axons or no axons at all exhibited myelination (Fig. 4(b-b")).

Structural analyses confirmed findings from immunofluorescence. Specifically, when examining semithin sections in the light microscope and ultrathin sections in the transmission electron microscope, we observed unmyelinated as well as myelinated axons in the LHBT (Fig. 5a-d). Unmyelinated axons were solely covered by Schwann cells and about $0.5 \mu \mathrm{m}$ in diameter. Myelinated axons were enwrapped by a myelin sheath which appeared in dark blue color in semithin sections and in black color in ultrathin sections. The thickness of myelinated axons including the myelin sheath was about $2 \mu \mathrm{m}$ (Fig. 5b, d). Unmyelinated and myelinated axons were grouped in fascicles which were outlined by a perineural sheath (Fig. 5b).

\section{Axons exhibit CGRP, substance $P$, TH, and GAP43 immunoreactivity}

In a next series of experiments, we analyzed the molecular quality of axons in the LHBT and performed immunolabeling with antibodies against CGRP, SP, TH, and GAP43. Nerve fibers were counterstained with anti-neurofilament. CGRP and SP are neuropeptides in nociceptive fibers. TH is found in sympathetic, noradrenergic neurons and GAP43 is a protein which is expressed in axons during development and regeneration.

For visualization of putative nociceptive fibers, three staining combinations were performed: (i) anti-neurofilament/anti-CGRP, (ii) anti-neurofilament/anti-SP, and (iii) anti-CGRP/anti-SP. In the LHBT, we observed neurofilament-positive axons that expressed CGRP and SP (Fig. 6(a-a", b-b")). However, a proportion of neurofilament-positive axons lacked CGRP/SP immunoreactivity indicating that axonal components with non-nociceptive quality are also present in LHBT (Fig. 6(a-a", b-b")). Double labeling with anti-CGRP/anti-SP revealed nerve fascicles where all axons coexpressed CGRP and SP (Fig. 6(c- $\left.c^{6 ")}\right)$ ). We also observed other nerve fascicles where some axons coexpressed CGRP/SP while other axons expressed CGRP but not SP (Fig. $\left.6\left(d-d^{6}\right)\right)$. Most commonly, nerve fascicles were outside the adventitial layer of blood vessels. Only occasionally, single nociceptive axons were observed near the junction of the muscular and adventitial layer of arterioles (Fig. 8(c-c" $)$ ).

Sympathetic innervation was visualized by labeling with anti-neurofilament/anti-TH. Labeling showed that a high number of neurofilament-positive axons exhibited TH immunoreactivity. Other neurofilament-positive axons lacked $\mathrm{TH}$ immunoreactivity (Fig. 7(a-a", b-b“)). In few cases, we observed TH-positive axons which exhibited only a weak or no neurofilament signal. A possible explanation is that in these axons the neurofilament was at a concentration too low for antibody detection. TH-positive axons ran within nerve fascicles that accompanied vascular structures but most commonly
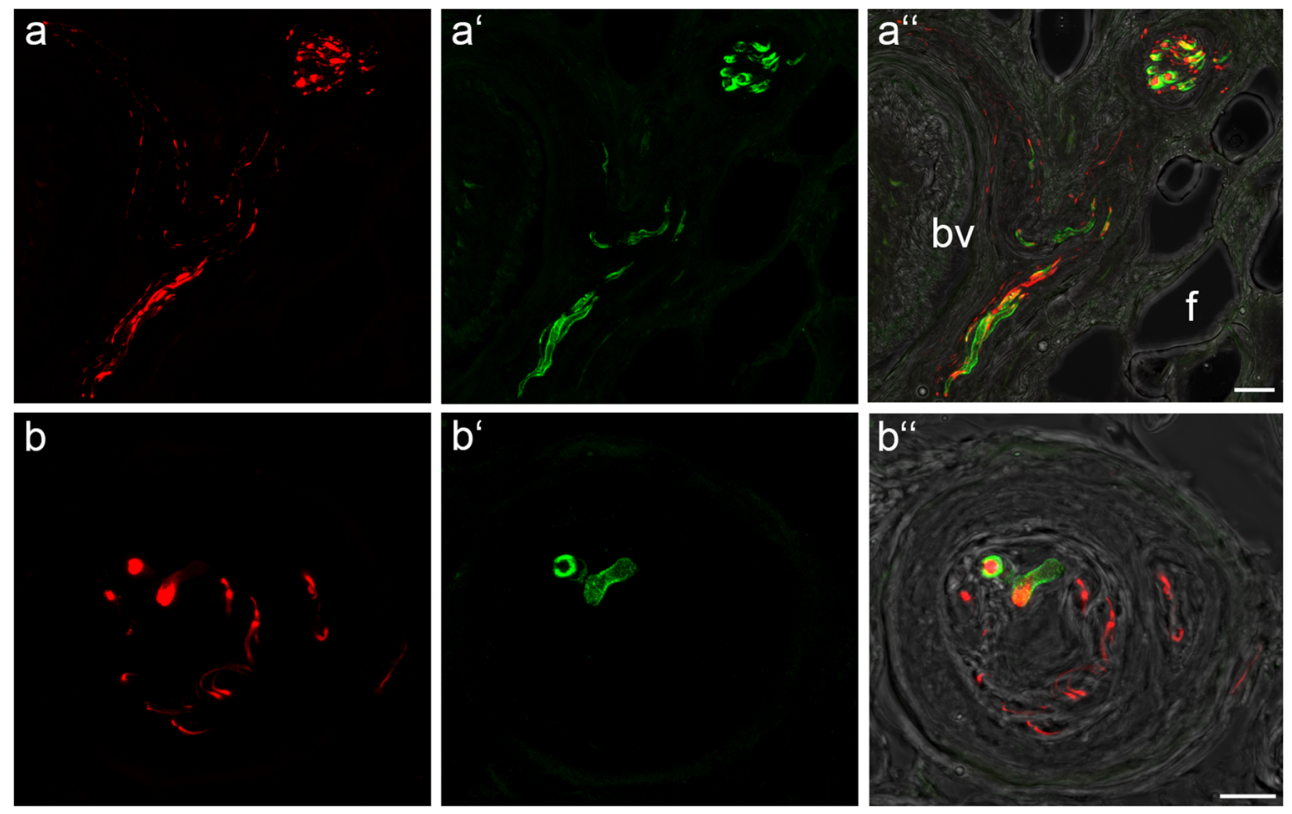

Fig. 4 CLSM images of sections labeled with anti-neurofilament and a marker for myelin (anti-MBP). (a, b) Nerve fibers are visualized by antineurofilament (red) and $\left(a^{6}, b^{c}\right)$ the myelin sheath by anti-MBP (green). Images in (b-b") are at high magnification. (a", b") Overlay showing myelinated as well as unmyelinated axons. Myelinated axons exhibit NF/MBP immunoreactivity, whereas unmyelinated axons are MBP-

negative. Myelinated axons have a larger diameter compared to unmyelinated axons. Bright field view showing cells with vacuole-like appearance thereby resembling fatty cells ( $\mathrm{f}$ in $\mathrm{a}^{\text {") }}$ ) and the anatomical structure of a nerve fascicle (b"). Blood vessel (bv). Scale bars, $20 \mu \mathrm{m}\left(\mathrm{a}-\mathrm{a}^{\circ}\right)$ and $10 \mu \mathrm{m}(\mathrm{b}-\mathrm{b} ")$ 
Fig. 5 Images from semithin and ultrathin sections. a Light microscopic view of a semithin section showing nerve fascicles containing myelinated as well as unmyelinated axons. Myelinated axons (arrows) are discernible due to dark blue color of the myelin sheath elicited through toluidine blue staining. Unmyelinated axons lack a dark blue envelop and are discernible by the white color of the axoplasm. Scale bar, $10 \mu \mathrm{m}$. b-d Images from the transmission electron microscope. b A nerve fascicle outlined by a perineural sheath and containing two myelinated axons (arrows) as well as unmyelinated axons (asterisks). Myelinated axons are surrounded by a black-colored myelin sheath which is formed by Schwann cells. Unmyelinated axons are embedded in Schwann cells but lack a myelin sheath. c High magnification of unmyelinated axons (highlighted in green color) embedded in a Schwann cell (s). Tenocyte (t). d Myelinated axon at high magnification
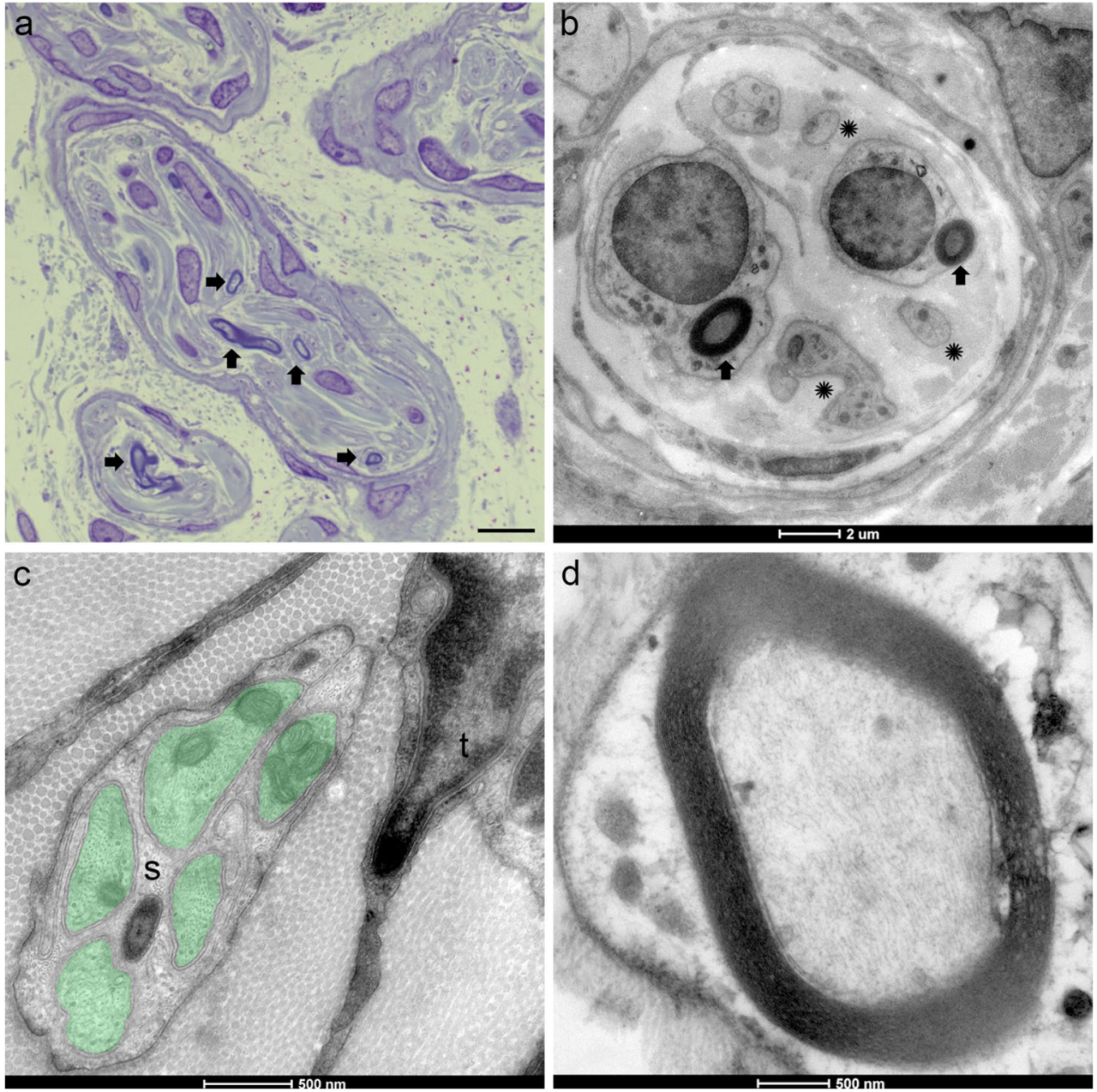

outside the adventitial layer. In other cases, TH-positive axons were found near the junction of the muscle layer and the adventitia of arteries (Fig. 7(b-b“)). TH-positive axons were of small diameter and exhibited varicosities along their length.

For GAP43 detection, two staining combinations were selected: (i) anti-neurofilament/anti-GAP43 and (ii) anti-SP/antiGAP43. Staining combination with anti-neurofilament/antiGAP43 exhibited that many neurofilament-positive axons in the LBTH expressed GAP43 as well. Exclusively, axons with larger diameter lacked GAP43 immunoreactivity (Fig. 8(a-a")). Staining combination with anti-SP/anti-GAP43 exhibited co-localization of SP and GAP43 in many axons (Fig. 8(b-b“, c-c"s)).

\section{CGRP is expressed in unmyelinated as well as myelinated axons, whereas SP, TH, and GAP43 are exclusively expressed in unmyelinated axons}

To verify which type of axon (unmyelinated versus myelinated) express CGRP, SP, TH, and GAP43, we performed additional immunolabeling experiments. Immunolabeling included (i) anti-CGRP/anti-MBP, (ii) anti-SP/anti-MBP, (iii) antiTH/anti-MBP, and (iv) anti-GAP43/anti-MBP. Anti-MBP visualized the myelin sheath of axons and was used to distinguish between myelinated and unmyelinated axons.

We observed CGRP immunoreactivity in unmyelinated as well as myelinated axons. Other myelinated axons lacked CGRP immunoreactivity (Fig. 9(a-a", b-b")). SP staining was confined to unmyelinated axons (Fig. 9(c-c")). TH expression was exclusively present in unmyelinated axon. This was an expected result since TH is an enzyme of postganglionic, unmyelinated sympathetic axons. TH-positive fibers ran often close to myelinated axons in nerve fascicles (Fig. 9(d-

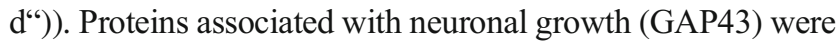
exclusively observed in unmyelinated axons (Fig. 9(e-e")). GAP43-positive unmyelinated axons were often located peripherally in nerve fascicles (Fig. 9(e-e" $)$ ) but were also observed more centrally where they intermingled with myelinated axons.

\section{Discussion}

This is the first comprehensive study that qualitatively characterized nervous elements in the proximal part of 

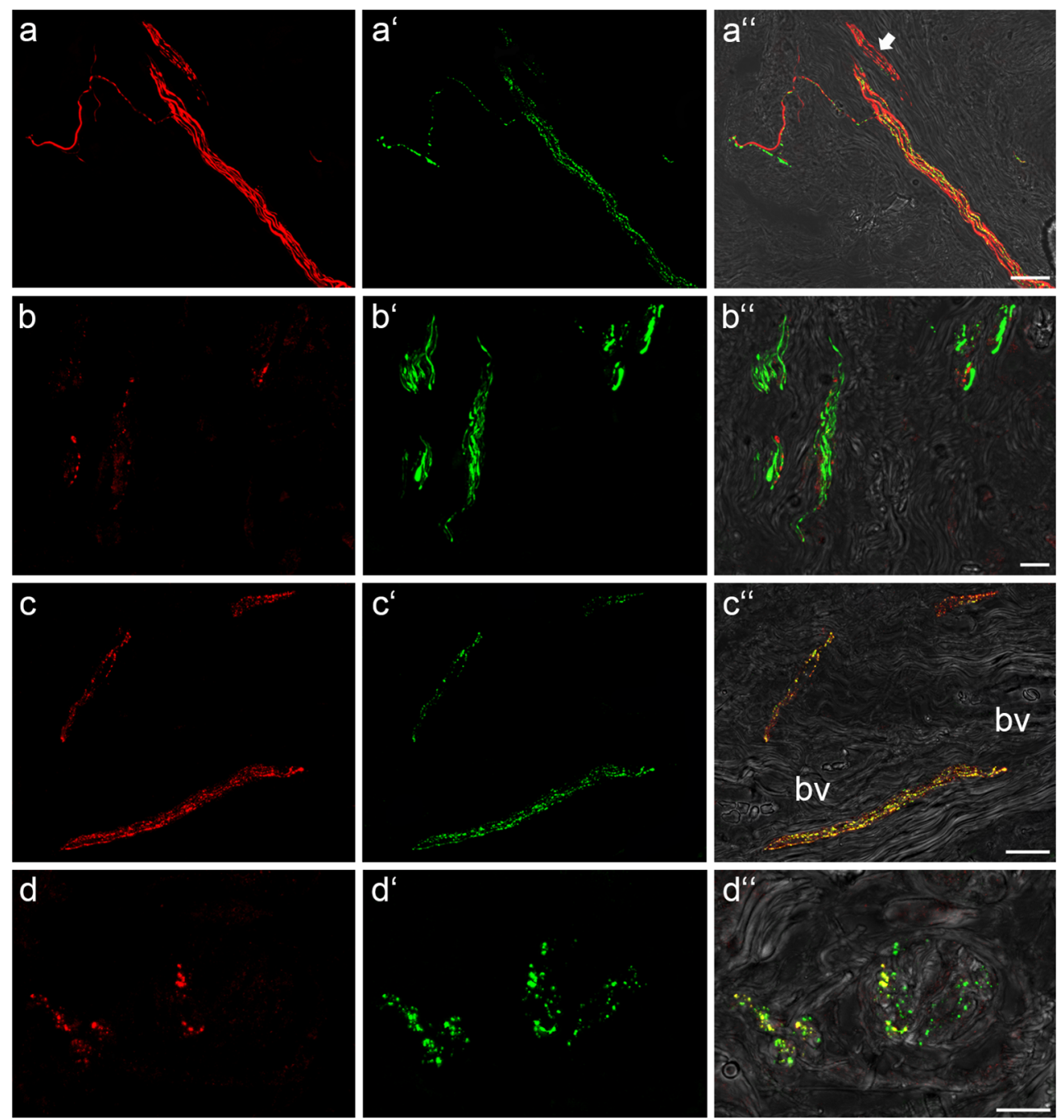

Fig. 6 CLSM images of sections labeled with a general neuronal marker (anti-neurofilament) and two selective markers for nociceptive nerve fibers (anti-CGRP and anti-SP). (a-a") Double labeling with antineurofilament (red) and anti-CGRP (green). (a) Axon visualized in neurofilament and $\left(a^{\circ}\right)$ CGRP staining. (a") Overlay showing that many axons exhibiting neurofilament immunoreactivity are CGRP-positive as well. Other neurofilament-positive axons (arrow) lack CGRP signal. Bright field view visualizes collagen fibers of the tendon. Scale bar, $25 \mu \mathrm{m}$. (b-b") Double labeling with anti-SP (red) and antineurofilament (green). (b) Axons visualized in SP and ( $\left.b^{6}\right)$ neurofilament staining. (b") Overlay showing that a minority of axons express SP/ neurofilament immunoreactivity and most neurofilament-positive axons lack SP. Bright field view visualizes collagen fibers of the tendon. Scale bar, $10 \mu \mathrm{m}$. (c-c"s, d-d") Double labeling with anti-SP (red) and antiCGRP (green). (c, d) Nerve fibers exhibiting SP and ( $c^{c}, d^{\star}$ ) CGRP immunoreactivity. (c", d") Overlay showing complete overlap of SP/CGRP immunoreactivity in c" and only partial overlap of SP/CGRP in (d"). Bright field view visualizes blood vessels (bv) and collagen fibers of the tendon $\left(\mathrm{c}^{\text {") }}\right)$ and the anatomical structure of a nerve fascicle $\left(\mathrm{d}^{\text {") }}\right)$. Scale bars, $20 \mu \mathrm{m}\left(\mathrm{c}-\mathrm{c}^{\text {"c }}\right)$ and $10 \mu \mathrm{m}\left(\mathrm{d}-\mathrm{d}^{\text {“ }}\right)$ the LHBT. We show that the LHBT is richly innervated with unmyelinated as well as myelinated nerve fibers which group in nerve fascicles and follow blood vessels. Manny myelinated nerve fibers and a subpopulation of unmyelinated fibers exhibit molecular features of putative nociceptive nerve fibers. Another subpopulation of unmyelinated nerve fibers exhibits molecular characteristics of sympathetic nerve fibers. Unmyelinated nerve fibers express proteins which are found during development and regeneration. Figure 10 summarizes the results of the present study.

\section{Unmyelinated as well as myelinated axons group in nerve fascicles close to blood vessels}

Two general neuronal markers (anti-neurofilament and antiPGP9.5) visualized the overall innervation of the LHBT. In accordance with previous studies, we observed a high number of nerve fibers in the LHBT which had a linear appearance and grouped in nerve fascicles in the vicinity blood vessels but outside the adventitial layer (Blumer et al., 2019, Boesmueller et al., 2017, Schmalzl et al., 2019, Singaraju et al., 2008). In arteries but not in veins, we observed single nerve fibers near the junction of 
Fig. 7 CLSM images of sections labeled with a general neuronal marker (anti-neurofilament) and a marker for sympathetic axons (anti-TH). (a, b) Axons visualized in NF staining (red) and $\left(a^{a}, b^{c}\right)$, TH staining (green). (a“, $\left.b^{\prime \prime}\right)$ Overlay showing axons that exhibit NF/TH immunoreactivity, whereas other NF-positive axons lack TH. Bright field views visualize the anatomical structure of blood vessels (bv) and collagen fibers. NF/TH immunoreactive axons in the vicinity of the blood vessels are outside the adventitial layer as well as near the muscleadventitia junction (arrow, b"). Scale bars, $50 \mu \mathrm{m}\left(\mathrm{a}-\mathrm{a}^{\prime \prime}\right)$ and $20 \mu \mathrm{m}\left(\mathrm{b}-\mathrm{b}^{\prime \prime}\right)$
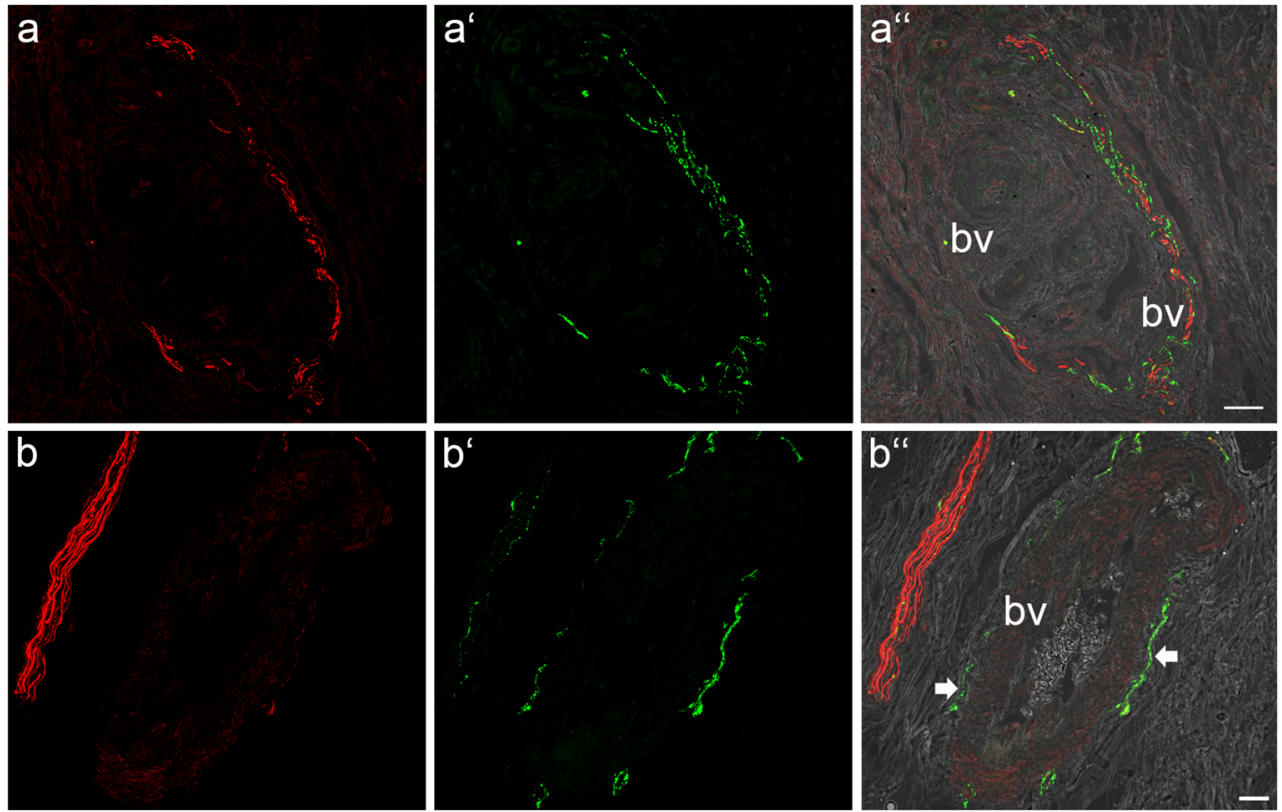

the muscular and the adventitial layer. Heterogeneity in perivascular nerve fiber distribution has been previously described in the tendon of the human extensor carpi radialis brevis muscle and blood vessels with and without innervation have been observed (Ljung et al., 1999b). Probably, in analogy to present findings, this disparity in vascular innervation depends on the type of blood vessel, i.e., artery versus vein. In a previous study of the cat Achilles tendon, nerve fibers close to venous and lymphatic vessels have been found (Andres et al., 1985). In the present study, nerve fibers were exclusively associated to vascular structures with a prominent muscle layer as demonstrated by phalloidin labeling, thereby confirming the arterial phenotype of
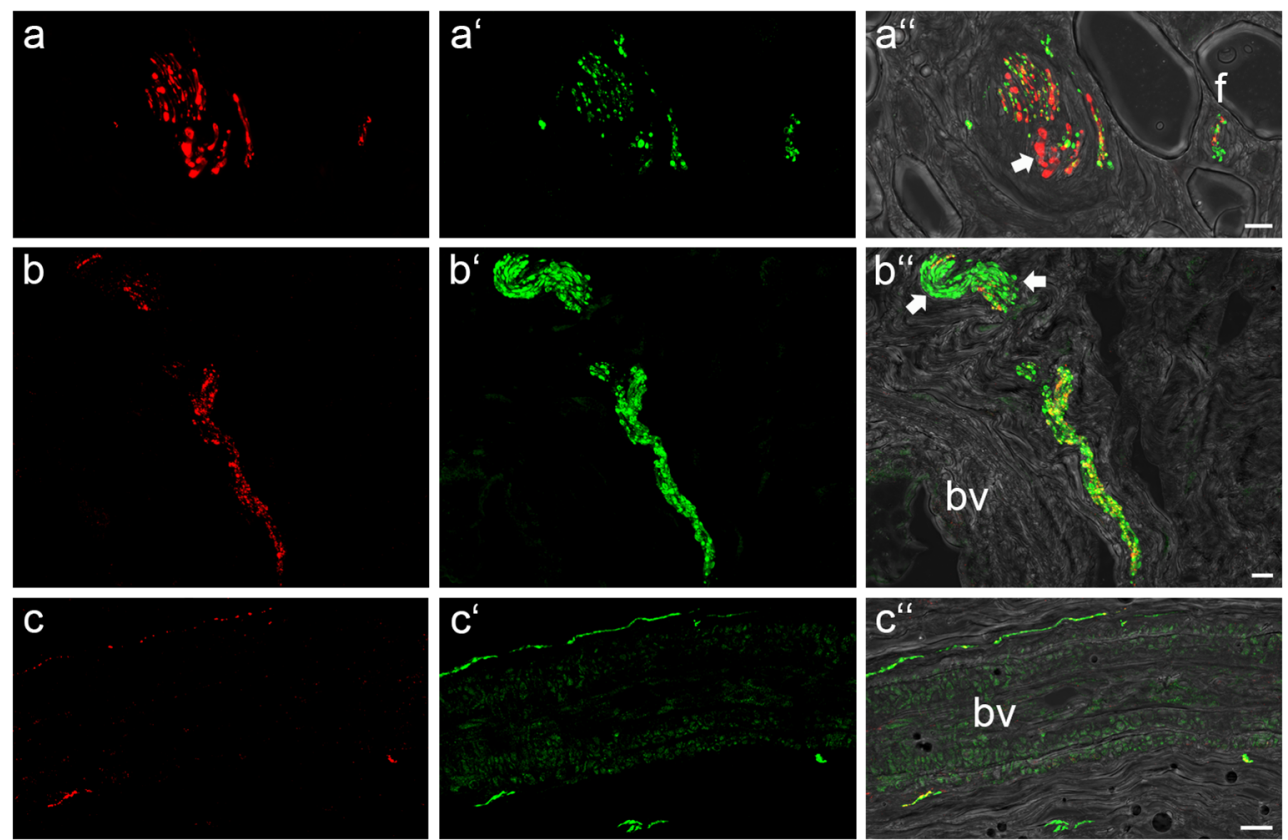

Fig. 8 CLSM images of sections labeled with a marker for neuronal development (anti-GAP43) in combination with anti-neurofilament and anti-SP. (a-a") Double labeling with anti-neurofilament (red) and antiGAP43 (green). (a) Axons visualized in NF and (a') GAP43 staining. (a") Overlay showing that many axons express NF and GAP43. Largediameter axons (arrow) lack GAP43 immunoreactivity. Bright filed view shows cell with vacuole-like appearance thereby resembling fatty cells (f). Scale bar, $10 \mu \mathrm{m}$. (b-b“, c-c") Double labeling with anti-SP (red) and
anti-GAP43 (green). (b, c) Axons visualized in SP and (b', c c) GAP43 staining. (b", $c^{\text {") }) ~ O v e r l a y s ~ e x h i b i t i n g ~ c o-l o c a l i z a t i o n ~ o f ~ S P ~ a n d ~ G A P 43 . ~}$ Some GAP43 positive axons (arrows) lack SP-immunoreactivity. Bright field view showing collagen fibers and a part of a blood vessels (bv) in orthogonal view (b") and longitudinal view (c"). In (c"), immunoreactive axons are near the muscle-adventitia junction. Scale bars, $10 \mu \mathrm{m}\left(\mathrm{b}-\mathrm{b}^{\circ}\right)$ and $20 \mu \mathrm{m}\left(\mathrm{c}-\mathrm{c}^{\prime \prime}\right)$ 

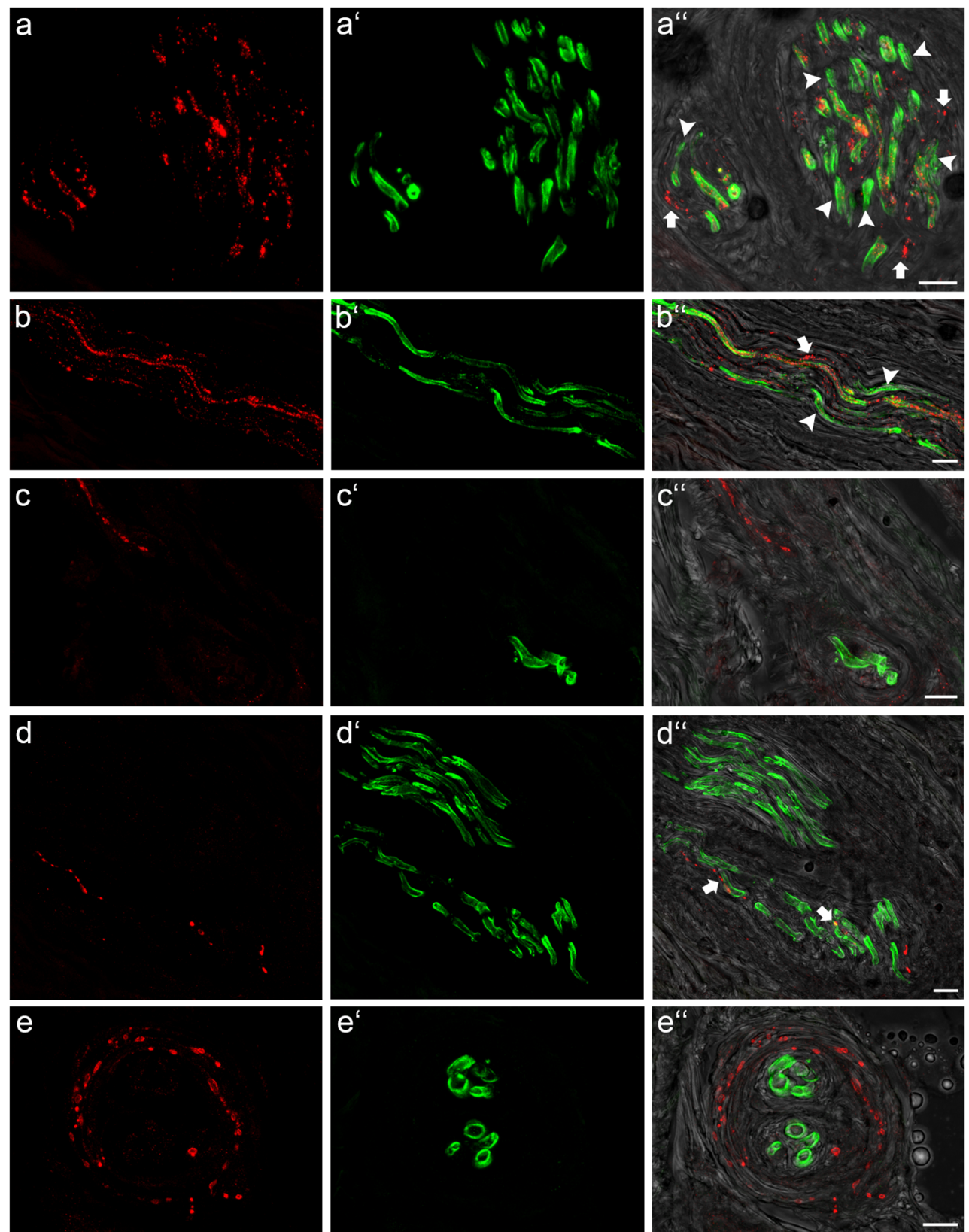

Fig. 9 CLSM images of sections labeled with a marker for myelinated axons (anti-MBP) in combination with anti-CGRP, anti-SP, anti-TH, and anti-GAP43. (a-a", b-b") Double labeling with anti-CGRP (red) and antiMBP (green). (a-a") Axons visualized in orthogonal and $\left(b-b^{\prime \prime}\right)$ in longitudinal view. (a, b) Showing axons positive for CGRP. ( $\left.a^{6}, b^{\prime}\right)$ Myelination is visualized by anti-MBP. (a", b") Overlay showing some axons expressing CGRP and MBP. Other axons express MBP but lack CGRP immunoreactivity (arrowheads). Other CGRP-positive axons lack myelin (arrows). Bright field views showing the anatomical structure of nerve fascicles in orthogonal view (a") and longitudinal view (b"). Scale bars, $10 \mu \mathrm{m}$. (c-c") Double labeling with anti-SP (red) and anti-MBP (green). (c) Axons positive for SP and ( $\left.c^{c}\right)$ axons positive for MBP. SP-

the blood vessels. We did not observe nervous elements in the adventitia of thin-walled vascular structures like venous and lymphatic vessels. This lack of innervation may indicate differences between species. immunoreactive axons lack a myelin sheath as shown in the overlay $\left(\mathrm{c}^{\text {") }}\right.$. Bright field shows collagen fibers. Scale bar, $10 \mu \mathrm{m}$. (d-d") Double labeling with anti-TH (red) and anti-MBP (green). (d) Showing axons positive for TH and (d`) axons positive for MBP. (d“) TH-positive axons are MBP negative. Arrows indicate TH-positive axons very close to myelinated nerve fibers. Bright field shows the anatomical structure of a nerve fascicle. Scale bar, $10 \mu \mathrm{m}$. (e-e") Double labeling with antiGAP43 (red) and anti-MBP (green). (e) Axons positive for GAP43 and $\left(\mathrm{e}^{6}\right)$ axons positive for MBP. All GAP43-immunoreactive axons are MBP negative (e"). Bright field shows the anatomical structure of a nerve fascicle. Scale bar, $10 \mu \mathrm{m}$

Besides unmyelinated axons, a substantial proportion of axons in the LHBT exhibited myelination as demonstrated by immunolabeling for myelin basic protein and structural analyses. Myelinated nerve fibers were observed in each 
Fig. 10 A summary of the results of the present study. (a) Showing the LHBT in its anatomical context and (b) the most proximal part of the LHBT analyzed in the present study. (c) Inside view of the tendon showing nerve fascicles (yellow) accompanying blood vessels (bv). (d) Detailed view of a nerve fascicle consisting of myelinated and unmyelinated axons. Axons with a myelin sheath are highlighted by a green circumference. Myelinated axons expressing CGRP are highlighted in green/orange color. A subpopulation of unmyelinated axons expresses TH (blue) and GAP43 (yellow) and is highlighted in blue/yellow color. Another subpopulations of unmyelinated axons expresses CGRP (orange), SP (dark red), and GAP43 (yellow). They are highlighted in orange/dark red/yellow color

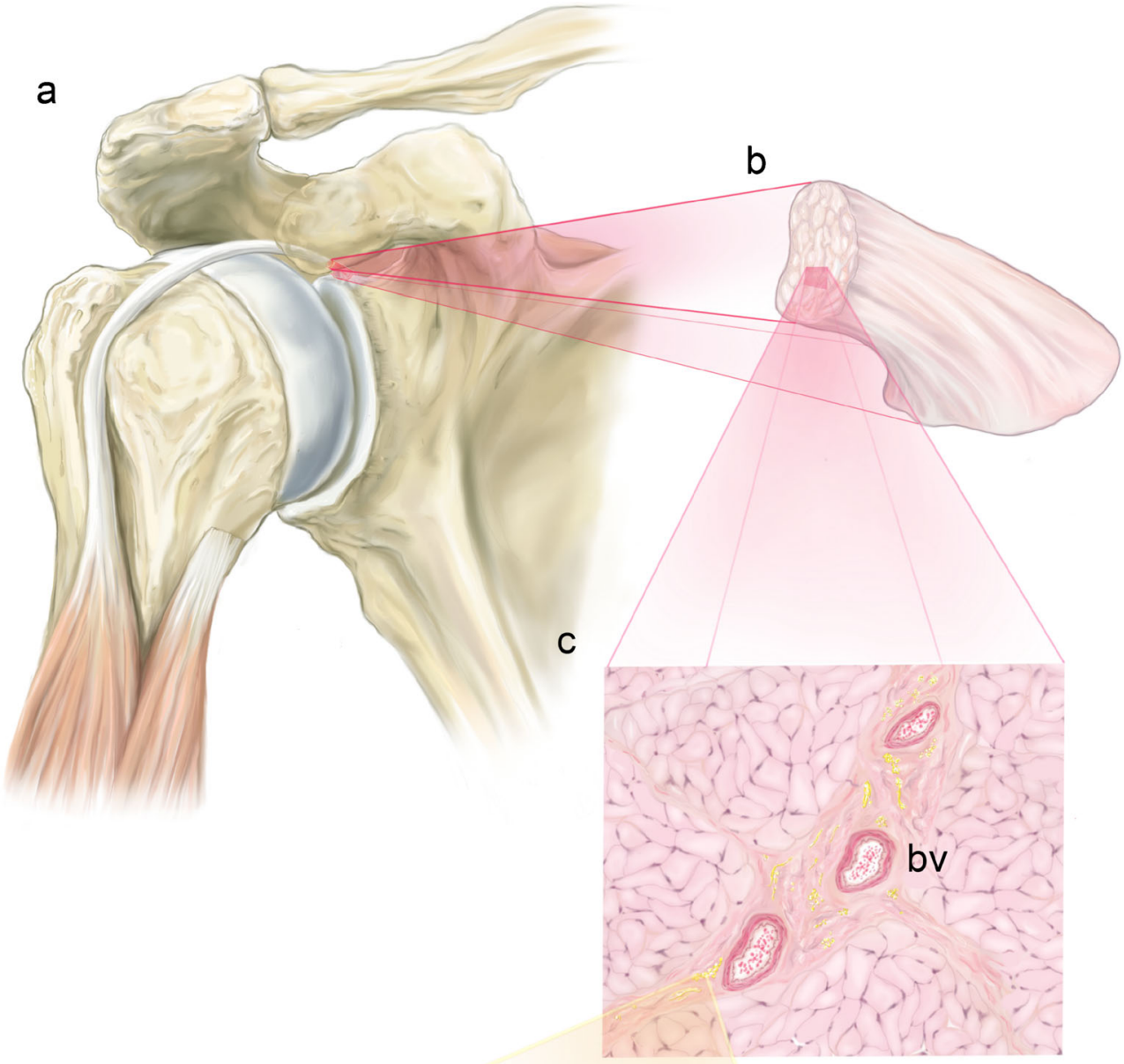

d

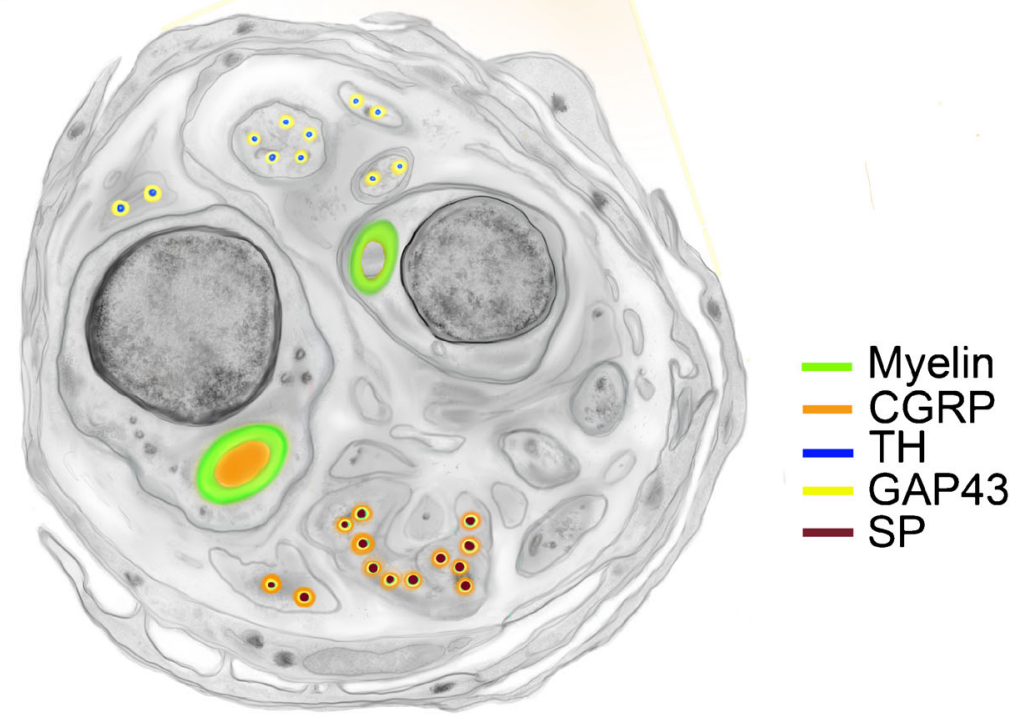

specimen suggesting that they are a regular constituent of LHBT. Meyelinated nerve fibers have been observed in the Achilles tendon of cat (Andres et al., 1985) but so far not in human tendons including the LHBT (Alpantaki et al., 2005, Boesmueller et al., 2017, Schmalzl et al., 2019), Achilles tendon (Ackermann et al., 2003,
Alfredson et al., 2003), plantaris tendon (Spang et al., 2015), and proximal tendon of the flexors and extensors of the forearm (Ljung et al., 2004, Ljung et al., 1999a). Thus, our novel findings would prompt for further analyses to clarify whether myelination is a specific feature of the LHBT or is repeated in other human tendons. 


\section{Putative nociceptive fibers including myelinated $A \delta$ and unmyelinated C-fibers are present in LHBT}

CGRP and SP are neuropetides which are present in nociceptive fibers. By double labeling with a general neuronal marker (anti-neurofilament) and selective markers for nociceptive axons (anti-CGRP and anti-SP), we demonstrated that a subpopulation of axons in the LHBT expressed CGRP and SP. CGRP/SP immunoreactivity has been previously described in the LHBT (Singaraju et al., 2008, Alpantaki et al., 2005), Achilles tendon (Ackermann et al., 1999, Ackermann et al., 2003, Andersson et al., 2008, Bjur et al., 2005), plantaris tendon (Spang et al., 2015), and proximal tendon of the extensor carpi radialis brevis (Ljung et al., 2004, Ljung et al., 1999b, Ljung et al., 1999a). Interestingly, there are some discrepancies regarding CGRP/SP distribution between present findings and two prior studies on the LHBT (Alpantaki et al., 2005, Singaraju et al., 2008). Specifically, Singaraju et al. (2008) observed CGRP/SP in neuronal as well as in nonneuronal elements like blood vessels, whereas present finding show that CGRP/SP immunoreactivity was exclusively confined to nervous elements. Using sequential immunolabeling on consecutive sections, Alpantaki et al. (2005) has suggested complete overlap of neurofilament and CGRP/SP in the LHBT. By simultaneous immunolabeling which is more conclusive, we observed only partial overlap of CGRP/SP and neurofilament indicating that only a subpopulation of axons transmits pain. A higher level of CGRP compared to SP has been previously described in the human Achilles tendon suggesting not complete co-localization of both neuropeptides (Bjur et al., 2005). This was confirmed in the present study. Specifically, double labeling with anti-CGRP/anti-SP showed that many axons exhibited CGRP/SP immunoreactivity, whereas other axons expressed CGRP alone.

Based on myelination, two major classes of nociceptive fibers can be distinguished: small-diameter unmyelinated Cfibers and larger diameter myelinated $A \delta$-fibers (Basbaum et al., 2009). To verify whether these classes of nociceptive axons are present in the LHBT, immunolabeling with antibody against myelin basic protein (MBP) in combination with CGRP and SP was performed. We observed CGRP immunoreactivity in MBP-negative axons (unmyelinated axons) and in many MBP-positive axons (myelinated axons), whereas SP immunoreactivity was confined to MBP-negative unmyelinated axons (see Fig. 10). Thus, present findings suggest that CGRP/SP-positive unmyelinated axons typify putative Cnociceptive fibers and CGRP-positive myelinated axons typify putative $\mathrm{A} \delta$-nociceptive fibers. Nociceptive fibers have been previously described in different human tendons but this is the first study that demonstrated that both classes of nociceptive fibers, namely, unmyelinated $\mathrm{C}$-fibers and myelinated $\mathrm{A} \delta$-fibers are present. $\mathrm{A} \delta$-fibers conduct nerve impulses fairly quickly and give way to the first, well-localized acute pain, whereas C-fibers have a slower conduction velocity and give way to the second, poorly localized dull pain which lasts for a longer period of time. Present findings demonstrate that the LHBT has the neuroanatomical substrate for transmitting acute as well as dull pain. Our findings are in line with a previous electrophysiological study of the cat Achilles tendon where group III myelinated axons (A $\delta$-fibers) and group IV unmyelinated axons (C-fibers) responding to noxious stimuli have been found (Mense and Meyer, 1985). Besides that, nerve fibers responding to temperature and mechanical stimuli have been found in the cat Achilles tendon (Mense and Meyer, 1985). At this point, it is worthy to mention that $A \delta$-fibers and C-fibers have also been found in other joint-associated structures like the knee joint capsule of human (Halata et al., 1985) and cat (Heppelmann et al., 1995).

\section{Sympathetic innervation of the LHBT might contribute to pain sensation}

Sympathetic nerve fibers have been found in the proximal tendon of the extensor carpi radial brevis (Ljung et al., 1999b), the patellar tendon (Danielson et al., 2007), and the Achilles tendon (Ackermann et al., 2001). Concordant with these studies (Ackermann et al., 2001, Danielson et al., 2007, Ljung et al., 1999b), we found TH-immunoreactive sympathetic nerve fibers are always close to blood vessels either outside the adventitial layer or close to the media-adventitia junction. So far, there is only one study that has reported on sympathetic innervation of the LHBT without, however, providing image information (Alpantaki et al., 2005). Different to our findings, Alpantaki et al. (2005) has reported that sympathetic fibers are not associated with vascular structures. Besides postganglionic sympathetic fibers expressing TH, non-neural elements (tenocytes) expressing TH have been found in the patellar tendon (Danielson et al., 2007) and Achilles tendon (Bjur et al., 2008). Moreover, TH expression was increased in tenocytes of tendinopathic compared to healthy tendons. In the present study, LHBTs were obtained from aged persons suffering from arthritis or had a nonreconstructable head split fracture and age-related alterations are most likely. In analogy to findings in the patellar and Achilles tendon, someone could expect that tenocytes in the LHBT express TH as well. However, different to Bjur et al. (2008)and Danielson et al. (2007)), we did not observe tenocytes expressing TH. TH exclusively co-localized with nervous elements as demonstrated by labeling with anti-neurofilament/anti-TH. At the moment, the variations of TH immunoreactivity in tenocytes of different tendons are inexplicable.

Besides its traditional effector function, several data suggest that the sympathetic nervous system is involved in amplifying and maintaining painful sensations and this is accomplished via functional interactions with sensory nociceptive 
fibers (Roberts and Elardo, 1985, Raja, 1995, Zamuner et al., 2015, Dawson et al., 2011). In the patellar tendon, $\alpha 1$ adrenergic receptors have been described in nerve fascicles that showed immunoreactivity for TH and SP suggesting that noradrenalin released from sympathetic terminals target adrenergic receptors on nociceptive fibers (Danielson et al., 2007, Dawson et al., 2011). In the LHBTs of the present study, sympathetic axons ran very close to myelinated axons which can express CGRP (putative A $\delta$-nociceptive fibers) suggesting sympathetic-nociceptive coupling. It is thus possible that efferent sympathetic nerve fibers communicate with afferent nociceptive fibers to augment pain sensations.

\section{GAP43 expression suggests neoinnervation in the LHBTs}

There is evidence that tendinopathic tendons exhibit a higher axonal density compared to healthy tendons suggesting that tendon disorders are associated with nerve ingrowth (Bjur et al., 2005, Ackermann et al., 2003, Alfredson et al., 2003). Accordingly, a single study has demonstrated that the GAP43 expression which is associated with neuronal growth is significantly higher in tendinopathic rotator cuff tendons compared to healthy control tendons (Xu et al., 2011). To verify neoinnervation in tendons of the present study, we analyzed the GAP43 expression. For ethical reasons, LHBTs of young persons were not available and it was not possible to compare neoinnervation in tendons of young and aged persons. In accordance with Xu et al. (2011), we observed neurofilament/GAP43positive axons in the vicinity of blood vessels. GAP43-positive nerve fibers lacked a myelin sheath and expressed SP as demonstrated by anti-GAP43/anti-MBP and anti-GAP43/anti-SP immunolabeling. Thus, based on GAP43 expression, the following conclusions are possible. First, neoinnervation in the LHBT involves unmyelinated but not myelinated nerve fibers. Second, newly formed unmyelinated nerve fibers include nociceptive Cfibers and sympathetic fibers (see Fig. 10). However, in this respect, it is worthy to mention that sympathetic axons are exceptional as they express GAP43 not only during growth but also in adulthood (Woolf et al., 1992). Third, since myelinated fibers do not express growth protein GAP43, myelinated axons including myelinated $A \delta$-nociceptive axons have to be interpreted as already existing fibers.

Ingrowth of nociceptive fibers has been observed in pathologic conditions including chronic pancreatitis and chronic back pain and increased quantity of SP/GAP43 correlates with sensation of pain (Freemont et al., 1997, Di Sebastiano et al., 1997). Tendon disorders are often painful and several data link neovascularization, neoinnervation, and chronic tendon pain (Alfredson et al., 2003, Ohberg et al., 2001, Schubert et al., 2005). This is the first study that demonstrates that neoinnervation involves putative nociceptive $\mathrm{C}$-fibers, thereby providing novel support that ingrowth of additional unmyelinated nociceptive fibers is the cause of chronic, dull pain in tendon disorders.

\section{Conclusion}

Here, we show that myelinated $\mathrm{A} \delta$-nociceptive fibers and unmyelinated C-nociceptive fibers coexist in the LHBT. Additionally, unmyelinated sympathetic fibers are present. $\mathrm{C}$-nociceptive fibers and sympathetic fibers express proteins which are found during neuronal development and neurorepair. The close vicinity of sympathetic and nociceptive fibers suggests sympathetic-nociceptive coupling which may augment pain sensation. Our neuroanatomical findings provide a basis to better understand the cause of acute and chronic pain in the LHBT.

Acknowledgments The authors wish to thank Regina Mayer, Marlene Rodler, and Astrid Haase for their helpful technical assistance.

Authors' contribution SB, RM, and RB designed the research. SB, RM, $\mathrm{BG}$, and $\mathrm{RB}$ performed the research and analyzed the data. $\mathrm{RB}$ wrote the manuscript. All authors made critical revision of the manuscript.

Funding Information Open access funding provided by Medical University of Vienna.

\section{Compliance with ethical statements}

Conflict of interest The authors declare that they have no conflict of interest.

Ethical approval All procedures performed in studies involving human participants were in accordance with the ethical standards of the institutional and/or national research committee and with the 1964 Helsinki declaration and its later amendments or comparable ethical standards. The study was approved by the local ethics committee (No. 06/2017).

Open Access This article is distributed under the terms of the Creative Commons Attribution 4.0 International License (http:// creativecommons.org/licenses/by/4.0/), which permits unrestricted use, distribution, and reproduction in any medium, provided you give appropriate credit to the original author(s) and the source, provide a link to the Creative Commons license, and indicate if changes were made.

\section{References}

Ackermann PW, Finn A, Ahmed M (1999) Sensory neuropeptidergic pattern in tendon, ligament and joint capsule. A study in the rat. Neuroreport 10:2055-2060

Ackermann PW, Li J, Finn A, Ahmed M, Kreicbergs A (2001) Autonomic innervation of tendons, ligaments and joint capsules. A morphologic and quantitative study in the rat. J Orthop Res 19:372-378

Ackermann PW, Li J, Lundeberg T, Kreicbergs A (2003) Neuronal plasticity in relation to nociception and healing of rat Achilles tendon. $\mathrm{J}$ Orthop Res 21:432-441 
Ahrens PM, Boileau P (2007) The long head of biceps and associated tendinopathy. J Bone Joint Surg Br 89:1001-1009

Akesson E, Kjaeldgaard A, Seiger A (1998) Human embryonic spinal cord grafts in adult rat spinal cord cavities: survival, growth, and interactions with the host. Exp Neurol 149:262-276

Alfredson H, Lorentzon R (2000) Chronic Achilles tendinosis: recommendations for treatment and prevention. Sports Med 29:135-146

Alfredson H, Ohberg L, Forsgren S (2003) Is vasculo-neural ingrowth the cause of pain in chronic Achilles tendinosis? An investigation using ultrasonography and colour Doppler, immunohistochemistry, and diagnostic injections. Knee Surg Sports Traumatol Arthrosc 11: 334-338

Alpantaki K, McLaughlin D, Karagogeos D, Hadjipavlou A, Kontakis G (2005) Sympathetic and sensory neural elements in the tendon of the long head of the biceps. J Bone Joint Surg Am 87:1580-1583

Andersson G, Danielson P, Alfredson H, Forsgren S (2008) Presence of substance $\mathrm{P}$ and the neurokinin-1 receptor in tenocytes of the human Achilles tendon. Regul Pept 150:81-87

Andres KH, von During M, Schmidt RF (1985) Sensory innervation of the Achilles tendon by group III and IV afferent fibers. Anat Embryol (Berl) 172:145-156

Basbaum AI, Bautista DM, Scherrer G, Julius D (2009) Cellular and molecular mechanisms of pain. Cell 139:267-284

Bjur D, Alfredson H, Forsgren S (2005) The innervation pattern of the human Achilles tendon: studies of the normal and tendinosis tendon with markers for general and sensory innervation. Cell Tissue Res 320:201-206

Bjur D, Danielson P, Alfredson H, Forsgren S (2008) Immunohistochemical and in situ hybridization observations favor a local catecholamine production in the human Achilles tendon. Histol Histopathol 23:197-208

Blumer R, Boesmueller S, Gesslbauer B et al (2019) How to visualize the innervation pattern in tendons: a methodical guide. Ann Anat 225: $21-27$

Boesmueller S, Nogradi A, Heimel P et al (2017) Neurofilament distribution in the superior labrum and the long head of the biceps tendon. J Orthop Surg Res 12:181

Danielson P, Alfredson H, Forsgren S (2007) Studies on the importance of sympathetic innervation, adrenergic receptors, and a possible local catecholamine production in the development of patellar tendinopathy (tendinosis) in man. Microsc Res Tech 70:310-324

Danielson P, Andersson G, Alfredson H, Forsgren S (2008) Marked sympathetic component in the perivascular innervation of the dorsal paratendinous tissue of the patellar tendon in arthroscopically treated tendinosis patients. Knee Surg Sports Traumatol Arthrosc 16:621626

Dawson LF, Phillips JK, Finch PM, Inglis JJ, Drummond PD (2011) Expression of alpha1-adrenoceptors on peripheral nociceptive neurons. Neuroscience 175:300-314

Di Sebastiano P, Fink T, Weihe E et al (1997) Immune cell infiltration and growth-associated protein 43 expression correlate with pain in chronic pancreatitis. Gastroenterology 112:1648-1655

Freemont AJ, Peacock TE, Goupille P, Hoyland JA, O’Brien J, Jayson MI (1997) Nerve ingrowth into diseased intervertebral disc in chronic back pain. Lancet 350:178-181

Gesslbauer B, Hruby LA, Roche AD, Farina D, Blumer R, Aszmann OC (2017) Axonal components of nerves innervating the human arm. Ann Neurol 82:396-408

Halata Z, Rettig T, Schulze W (1985) The ultrastructure of sensory nerve endings in the human knee joint capsule. Anat Embryol (Berl) 172: 265-275

Heppelmann B, Messlinger K, Neiss WF, Schmidt RF (1995) Fine sensory innervation of the knee joint capsule by group III and group IV nerve fibers in the cat. J Comp Neurol 351:415-428

Kies MW, Martenson RE, Deibler GE (1972) Myelin basic proteins. Adv Exp Med Biol 32:201-214
Li JL, Wang D, Kaneko T, Shigemoto R, Nomura S, Mizuno N (2000) Relationship between neurokinin-1 receptor and substance $\mathrm{P}$ in the striatum: light and electron microscopic immunohistochemical study in the rat. J Comp Neurol 418:156-163

Ljung BO, Alfredson H, Forsgren S (2004) Neurokinin 1-receptors and sensory neuropeptides in tendon insertions at the medial and lateral epicondyles of the humerus. Studies on tennis elbow and medial epicondylalgia. J Orthop Res 22:321-327

Ljung BO, Forsgren S, Friden J (1999a) Substance P and calcitonin generelated peptide expression at the extensor carpi radialis brevis muscle origin: implications for the etiology of tennis elbow. J Orthop Res 17:554-559

Ljung BO, Forsgren S, Friden J (1999b) Sympathetic and sensory innervations are heterogeneously distributed in relation to the blood vessels at the extensor carpi radialis brevis muscle origin of man. Cells Tissues Organs 165:45-54

Maggi CA (1995) Tachykinins and calcitonin gene-related peptide (CGRP) as co-transmitters released from peripheral endings of sensory nerves. Prog Neurobiol 45:1-98

Mense S, Meyer H (1985) Different types of slowly conducting afferent units in cat skeletal muscle and tendon. J Physiol 363:403-417

Messner K, Wei Y, Andersson B, Gillquist J, Rasanen T (1999) Rat model of Achilles tendon disorder. A pilot study. Cells Tissues Organs 165: 30-39

Ohberg L, Lorentzon R, Alfredson H (2001) Neovascularisation in Achilles tendons with painful tendinosis but not in normal tendons: an ultrasonographic investigation. Knee Surg Sports Traumatol Arthrosc 9:233-238

Raja SN (1995) Role of the sympathetic nervous system in acute pain and inflammation. Ann Med 27:241-246

Roberts WJ, Elardo SM (1985) Sympathetic activation of unmyelinated mechanoreceptors in cat skin. Brain Res 339:123-125

Schmalzl J, Plumhoff P, Gilbert F et al (2019) The inflamed biceps tendon as a pain generator in the shoulder: a histological and biomolecular analysis. J Orthop Surg (Hong Kong) 27:2309499018820349

Schubert TE, Weidler C, Lerch K, Hofstadter F, Straub RH (2005) Achilles tendinosis is associated with sprouting of substance $\mathrm{P}$ positive nerve fibres. Ann Rheum Dis 64:1083-1086

Sethi N, Wright R, Yamaguchi K (1999) Disorders of the long head of the biceps tendon. J Shoulder Elb Surg 8:644-654

Singaraju VM, Kang RW, Yanke AB et al (2008) Biceps tendinitis in chronic rotator cuff tears: a histologic perspective. J Shoulder Elb Surg 17:898-904

Spang C, Harandi VM, Alfredson H, Forsgren S (2015) Marked innervation but also signs of nerve degeneration in between the Achilles and plantaris tendons and presence of innervation within the plantaris tendon in midportion Achilles tendinopathy. J Musculoskelet Neuronal Interact 15:197-206

Tosounidis T, Hadjileontis C, Triantafyllou C, Sidiropoulou V, Kafanas A, Kontakis G (2013) Evidence of sympathetic innervation and alpha1-adrenergic receptors of the long head of the biceps brachii tendon. J Orthop Sci 18:238-244

Woolf CJ, Reynolds ML, Chong MS, Emson P, Irwin N, Benowitz LI (1992) Denervation of the motor endplate results in the rapid expression by terminal Schwann cells of the growth-associated protein GAP-43. J Neurosci 12:3999-4010

$\mathrm{Xu}$ Y, Bonar F, Murrell GA (2011) Neoinnervation in rotator cuff tendinopathy. Sports Med Arthrosc Rev 19:354-359

Zamuner AR, Barbic F, Dipaola F et al (2015) Relationship between sympathetic activity and pain intensity in fibromyalgia. Clin Exp Rheumatol 33:S53-S57

Publisher's note Springer Nature remains neutral with regard to jurisdictional claims in published maps and institutional affiliations. 